\title{
The Consensus Formation in the Naming Game on Spatial Networks
}

\author{
Yiwei Liu, Lin Feng, Qiuhui Pan*, Linghui Tong, Kaiyu Zhan \\ School of Innovation and Entrepreneurship, Dalian University of Technology, Dalian 116024, China \\ ${ }^{*}$ Corresponding author
}

\begin{abstract}
The NG model is a model for the dissemination of opinions, focusing on the dissemination of mainstream opinions and how individuals move towards consensus in the adoption of a single opinion through discussion. This is a multi-individual model that uses exchange and negotiation between neighbors to share ideas, performing naming games without any central control on spatial networks. we consider the geometric structure in the two dimensions. In a $L^{*} \mathrm{~L}$ spatial grids, every grid represents an agent which owns his vocabulary, and every agent updates his vocabulary in terms of the word received from his neighbor. Finally all agents through the process of reaching an agreement. The agreement process can be divided into 3 stages. Firstly, the total number of words drop quickly and about 10 words left, and the remain words form several clusters, and the area of the clusters are almost the same. The adjacent agents in the same cluster have the same word. Secondly, the clusters mutually annex with the number of words slow decreasing. Because the word with the biggest area cannot gain momentum in the competition with other words with the similar area, the word with biggest area will be varying all the time. Thirdly, one area of word surpasses 50 percent and this area will be expanding continuously until reaching an agreement.
\end{abstract}

Keywords-naming game; interacting agent models; spatial networks

\section{BACKGROUND}

The NG model is a model for the dissemination of opinions, focusing on the dissemination of mainstream opinions and how individuals move towards consensus in the adoption of a single opinion through discussion.[1] This is a multi-individual model that uses exchange and negotiation between neighbors to share ideas. The model is conceived as an automatic language evolutionary system and plays an important role in studying the evolution of language through new words, new phrases appearing, and adoption of new meaning for different words.[2][3] This can be applied from the primary voting model of the artificial sensor network to opinion organizational model in social media[4].

At present, there are a lot of discussion about naming game in the world . Many interesting models have been built. They are used, for example, in the exploration of naming activities in new domains of agents and the sharing of lexicon in new network communication tools.

These models include Guo' article: Mainly for real society NG game simulation, they believe that when a point of view has been converged after any new idea is difficult to refute the original. The model is mainly based on the BA network, introducing the concept of freshness to measure the memory level of a viewpoint [5]. Adam Lipowski also mentions similar abrupt transition points in his paper [6]. The difference is that they explain this sudden change through the Bauer effect.

Luca Dall'asta's article: Unbalanced Naming Games Based on Complex Networks[7]; "turning point in the multi-individual system naming game based on the shared lexicon" by Andrea Baronchelli, published in the famous journal Science[8];

The two references cited above are leading-edge research in naming games, great reference significance. First, a Minimal model is proposed in[8]. a topic called symbolic dynamics is studied In[7], It deals mainly with how linguistic representations take place and evolve, and the spread is based only on basic inner rules and pairwise representations. And his research is sometimes based on the most original experiment - Talking Heads experiments[9], whose rules are free contact between individuals. It is clear that the case of a small number of participants is true, and there are potential topological rules [10], that is, in a low-lattice grid. The complex network of non-equilibrium game is to consider the condition of complex networks, that is, human contact is irregular; Consider complex topology, and will have an impact on the final agreement.

Committed nodes are assigned a commitment strength, $\mathrm{w}$, defining their willingness to lose (in waning), gain (for increasing) or both (in variable) commitment to an opinion. Such model has committed nodes that can stick to a single opinion for some time without losing their flexibility to change it in the long run. A change in commitment strength impacts the critical fraction of population necessary for a minority consensus. show that if different nodes have different values of $\mathrm{w}$, higher standard deviation of $\mathrm{w}$ increases the critical fraction for waning commitment and decrease this fraction for increasing commitment[11].

A model displays a non-equilibrium phase transition between an absorbing ordered consensus state, which occurs for small noise, and a disordered phase with fragmented clusters characterized by heterogeneous memories, which emerges at strong noise levels. The nature of the phase transition is studied by means of a finite-size scaling analysis of the moments. We observe a scaling behavior typical of a discontinuous transition and we are able to estimate the 
thermodynamic limit. The scaling behavior of the clusters size seems also compatible with this kind of transition. [12]

[13] Palombi study the transition to multi-language states in the stochastic block model and on networks with community overlap. We also examine the scaling of critical thresholds under variations of topological properties of the network, such as the number and relative size of communities and the structure of intra-/inter-community links.

[14] Pickering William find that the system with high Shannon entropy has a higher consensus time and a lower critical fraction of zealots compared to low-entropy states. We also show that the critical number of committed agents decreases with the number of opinions and grows with the community size for each word. our results suggest that committed minorities can more easily conquer highly diverse systems, showing them to be inherently unstable.

[15] Goles Eric introduce automata networks to model some features of the emergence of a vocabulary related with the naming game model. We study the dynamical behavior (attractors and convergence) of extreme and majority local functions

[16] Goles Eric analyse three models of opinion exchange that uncover the community structure of a network, based on the Naming Game (NG), a classic model of linguistic interactions of agreement. The NG-based models applied in this work insert time-changing social features to the NG dynamics in order to form communities of nodes sharing different language conventions. For this matter, we explore the models NG-AW-that incorporates trust-, NG-LEF-that incorporates uncertainty-and NG-SM-finally incorporating opinion preference.

[17] Lou Yang,study naming game with communication errors during pair-wise conversations, with error rates in a uniform probability distribution. First, a model of naming game with learning errors in communications (NGLE) is proposed. Then, a strategy for agents to prevent learning errors is suggested. To that end, three typical topologies of communication networks, namely random graph, small-world and scale-free networks, are employed to investigate the effects of various learning errors.

Our literature further model on the basis of the predecessors. Let $\mathrm{Nd}$ be the total number of unrelated words in all individual lexicon. The total number of words studied varied over time and we found the a plunge point of lexicon words. At the same time, we explain and demonstrate the plunge phenomenon in this lexicon: the final unification for which word is random. But, the word which is more used within a range will have opportunities to be used by more individuals. Individuals using the same word form a range in which the number of individuals is called the "area" of the range. Using the snapshot map to show the area of the word map can reflect the process of merging between words more clearly, and find the largest area of the law of change.

\section{MODEL}

As mentioned above in the introduction, first we consider the geometric structure in the two dimensions. In a $\mathrm{L}^{*} \mathrm{~L}$ spatial grids $(L=200)$, we supposed every grid represents an agent which own his vocabulary for a certain object. at the beginning, each agent own a name for the object, the name for the object of the agents is relevant to their own sequence number, from 1 to 40000. First, At the time t, every agent acting as a 'speaker' will transmit a word from his list of vocabulary at random to his neighbors. Second, every agent acting as a 'listener' update his vocabulary in terms of the word received from his neighbors. The basic algorithmic rules are as follows. A word from his neighbor's vocabulary will be chosen at random. If the listener has the word, the listener delete all other words, collapse the list of vocabulary to this one word. If the listener does not have the word transmitted by the speaker, he adds it to his vocabulary without any deletion .

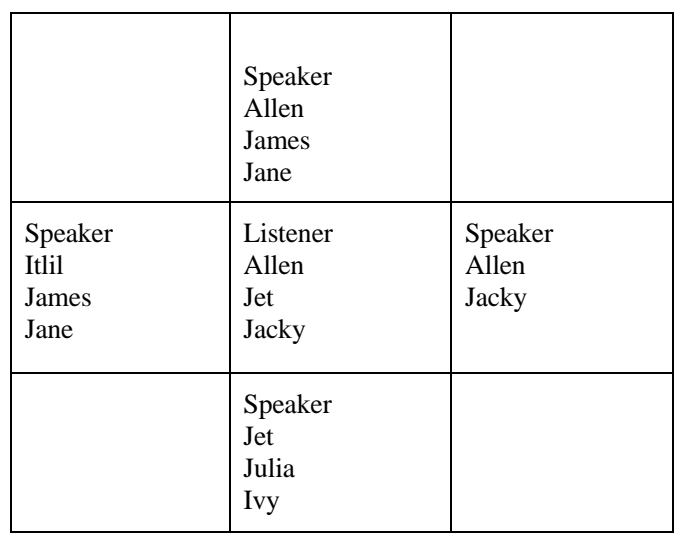

FIGURE I. THE RULE OF UPDATING VOCABULARY

In FIGURE I a word will be chosen at random from speak's vocabulary as the name to the object (taking the underlined word above for example), and listener also choose a word at random. In fact, although the the neighbor choose the word at random, due to different chosen words, the probability differs. The probability of James is 50 per cent while both Allen and jet are 25 percent.

If the chosen word is James, it will be added into the listener's vocabulary and the updating vocabulary is as follows:

\begin{tabular}{|l|}
\hline Listener \\
Allen \\
Jet \\
Jacky \\
James
\end{tabular}

If the word participating the game is Allen, only one word in listener's vocabulary remains and that is Allen. The updating vocabulary is as follows:

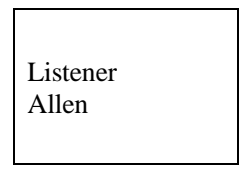

\section{RESUlT AND DISCUSSION}

$\mathrm{L}=200$, at the initial time, there is only one word as the name to object in the vocabulary of every agent. In this 
experiment we assume that the name is their own sequence number which from 1 to 40000. Trace the number of different words as $\mathrm{Nd}(\mathrm{t})$.

Time evolution of the $\mathrm{Nd}(\mathrm{t})$ in the naming game is as follows:

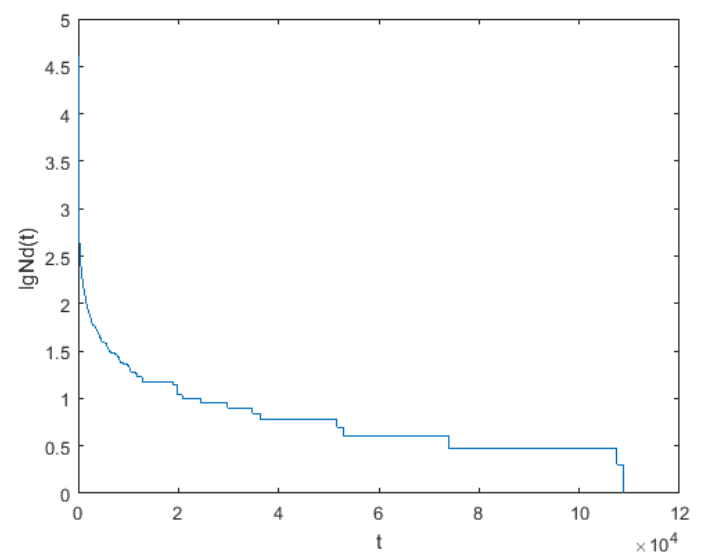

FIGURE II. TIME EVOLUTION OF THE NUMBER OF DIFFERENT WORDS IN THE NAMING GAME

As displayed above, following the early-time rapid decrease of the number of different words, the rate of decreasing numbers becomes a bit slow when about 10 words remain in the system. But eventually the agents vocabularies converge to a unique vocabulary shared among all agents which can be regarded as a unified name to new things. In the process of reaching an agreement, as the number of words decreases at express speed, it is obvious that the decreasing rate becomes slow when only a few words are left. Then we observe from the snapshots of the time evolution of the contents of the agents' word lists during the process of reaching an agreement.

Time evolution of the area of distribution of remaining words is as follows:
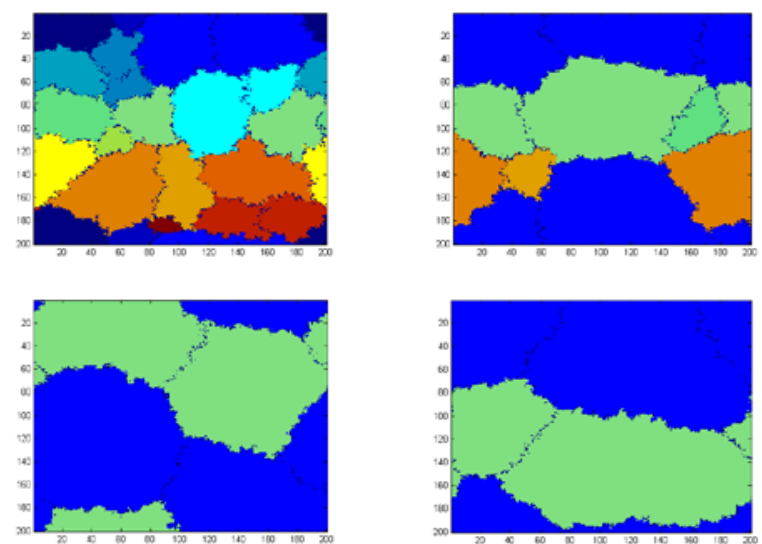
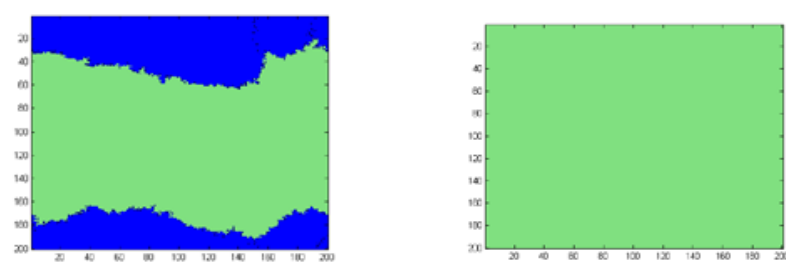

FIGURE III. THE PROVE OF REACHING AN AGREEMENT

As the FIGURE III demonstrated, a large number of small spatial clusters sharing the same name develop which means the local formation was established. Each color stand for the agent which have only one word, At the interfaces of small spatial clusters exists 'active' agents which have 2 or 3 words in their vocabularies and these words are in the neighboring clusters. These agents know about different names to the object and function as 'translators' to form a communication among the neighbors, in the meantime, with the evolution of time, by virtue of the translation which results in the slow coalescence of the interfaces separating the clusters, more and more of the small clusters are being eliminated, giving rise to the emergence of larger clusters, eventually leading to one cluster in which all agents sharing the same word.

In general, though the final word is at random, the word with a large many users in one cluster will have more chances to be utilized by more agents. Agents sharing the same word form a cluster and the number of agents in the cluster is defined as the 'area' of the cluster, trace the largest number of the cluster as $\mathrm{S}(\mathrm{t})$. As the FIGURE II displayed, following the early-time rapid increase of small clusters, words will 'annex' the words with relatively small area and the converging of the words with the similar areas will be a bit slow.

The biggest area in the system at every time step will be shown in the following.

Time evolution of biggest area is as follows:

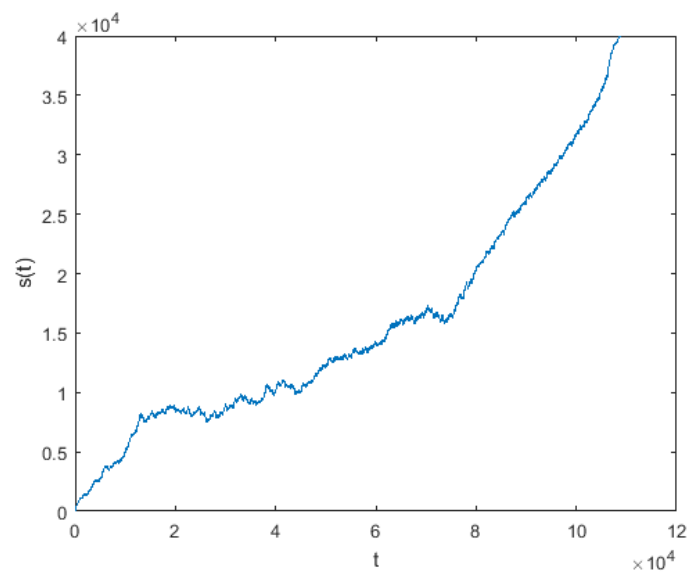

FIGURE IV.TIME EVOLUTION OF BIGGEST AREA

As FIGURE IV showed, the abscissa is time step and the ordinate is the biggest area in the system. 
At the early-time exists rapid increase of the biggest area of the words and the rapid decrease of the number of words, indicating that larger clusters expand its area by assimilating neighbors, and nearly 10 clusters with the similar size of the area stalemate when the area is up to the 30 percent of the total area. When the largest area surpasses 50 percent of the total area, it prevails, as a result, the area keeps expanding till reaching an agreement.

Trace the words with the biggest area as $\mathrm{w}(\mathrm{t})$,Time evolution of words with the biggest area is as follows:

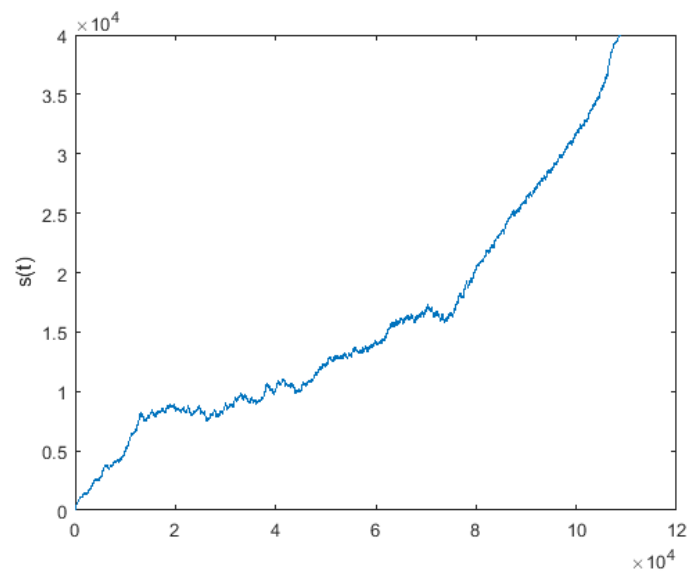

FIGURE V. TIME EVOLUTION OF NAME WITH THE BIGGEST AREA

As FIGURE V indicates, the abscissa is time step and the ordinate is name with the biggest area in the system as $\mathrm{R}(\mathrm{t})$ (at the early-time, the name to the object of the agents is relevant to their own sequence number, from 1 to 40000)

In the process of reaching an agreement, the word with the biggest area is varying constantly because the word with biggest area occupies the approximate proportion of other words and only by accounting for over 50 percent of total area, this word can form an absolute advantage until reaching an agreement. As the chart shows, the final sharing name is the 29676.

\section{SUMMARY}

Naming game in a $\mathrm{L} * \mathrm{~L}$ spatial grids, the process of reaching an agreement can be divided into 3 stages: First, the total number of words drop quickly and about 10 words left, and the remain words forms several clusters, and the area of the clusters are almost the same. the adjacent agents in the same cluster have the same word. Second, the clusters mutually annex with the number of words slow decreasing. Because the word with the biggest area cannot gain momentum in the competition with other words with the similar area, the word with biggest area will be varying all the time. Third, one area of word surpasses 50 percent and this area will be expanding continuously until reaching an agreement.

\section{ACKNOWLEDGEMENT}

We thank the anonymous Editors and Reviewers' warm work earnestly. The work is supported by the Fundamen-tal
Research Funds for the Central Universities (No. DUT16RC(4)85 ).

\section{REFERENCES}

[1] Maity, Suman Kalyan, Mukherjee, Animesh. Understanding how dominance affects the emergence of agreement in social network. Proceedings of 2012 ASE/IEEE International Conference on Privacy, Security, Risk and Trust and 2012 ASE/IEEE International Conference on Social Computing (SocialCom/PASSAT 2012). 2012: 508-513.

[2] Baronchelli Andrea, Felici Maddalena, Loreto Vittorio. Sharp transition towards shared vocabularies in multi-agent systems. JOURNAL OF STATISTICAL MECHANICS-THEORY AND EXPERIMENT. 2006-6: P06014.

[3] Steels Luc. A self-organizing spatial vocabulary. Artificial Life. 1995 (1996), 2(3): 319-332.

[4] Baronchelli Andrea. Role of feedback and broadcasting in the naming game. PHYSICAL REVIEW E. 2011-04-08, 83(4): 046103.

[5] Guo Dongwei, Meng Xiangyan, Liu Miao. Opinion alternating model and influence factors based on Naming Game. nternational Conference on Intelligent Human-Machine Systems and Cybernetics. 2011: 28-32.

[6] Lipowski Adam, Lipowska Dorota. Bio-linguistic transition and Baldwin effect in an evolutionary Naming-game model. INTERNATIONAL JOURNAL OF MODERN PHYSICS C. 2008-03, 19(3): 399-407.

[7] Luca Dall'Asta, A. Barrat, V. Loreto. Physical Rev E. 2006-10.

[8] A. Baronchelli, M. Felici, E. Caglioti, V. Loretoand L. Steels. Preprint, arxiv. Physics. 2005: 0509075.

[9] L. Steels. Autonomous Agents and Multi-Agent Systems 1, the talking heads experiment, Volume 1. Words and Meaning, Laboratorium, Antwerpen(Belgium). 1998: 169-194.

[10] A. Baronchelli, L. Dall'Asta, A. Barrat, V. Loreto. Phys.Rev.E. 2006, 73: 015102(R).

[11] Niu, X, Doyle, C, Korniss, G, Szymanski, BK. The impact of variable commitment in the Naming Game on consensus formation. SCIENTIFIC REPORTS 7. 2012-2: 41750.

[12] Brigatti E., Hernandez A. Finite-size scaling analysis of a nonequilibrium phase transition in the naming game model. PHYSICAL REVIEW E. 2016-11-10, 94(5): 052308.

[13] Palombi, F., Toti, S. Topological aspects of the multi-language phases of the Naming Game on community-based networks [arXiv] arXiv. 2016-10-27: 39.

[14] Pickering William, Szymanski Boleslaw K., Lim Chjan. Analysis of the high-dimensional naming game with committed minorities. PHYSICAL REVIEW E. 2016-05-25, 93(5): 052311.

[15] Goles Eric, Montealegre Pedro, Vera Javier. Naming Game Automata Networks. JOURNAL OF CELLULAR AUTOMATA. 2016, 11(5-6): 497-521.

[16] Goles Eric, Montealegre Pedro, Vera Javier. Naming Game Automata Networks. JOURNAL OF CELLULAR AUTOMATA. 2016, 11(5-6): 497-521.

[17] Lou Yang, Chen Guanrong. Analysis of the "naming game" with learning errors in communications. SCIENTIFIC REPORTS, 2015-07-16, 5: 12191. 